\title{
LA INCORPORACIÓN DE LA MEDICINA DE FAMILIA EN LA ENSEÑANZA DE LA MEDICINA EN ESPAÑA
}

\author{
THE INCLUSION OF FAMILY MEDICINE IN MEDICAL EDUCATION IN SPAIN
}

\begin{abstract}
Ángel Otero
Director del Centro Universitario de Salud Pública (CUSP) y Coordinador de la Unidad de Atención Primaria de la Universidad Autónoma de Madrid (UAM)
\end{abstract}

A lo largo de la última década (1991-2000) y coincidiendo con el especial protagonismo que la Atención Primaria tuvo en la reforma sanitaria en España, la mayoría de las Facultades de Medicina españolas han ido incorporando como novedad en su currículum actividades relacionadas con la Medicina de Familia y la Atención Primaria ${ }^{(1)}$.

A pesar de esta incorporación generalizada, estas actividades aún no han llegado a ser asumidas como auténticamente importantes por el cuerpo docente de las Facultades, apareciendo en la mayoría de los nuevos programas como actividades de segunda fila sin una integración real en las asignaturas troncales que definen clásicamente la carrera de medicina.

La aparición de estas actividades en el seno de la universidad se debe mas a la influencia de los grandes cambios que se están produciendo en el sistema sanitario español, y más concretamente en la organización del nivel de la atención primaria, que no a un impulso nacido en las propias Facultad de Medicina. Se corresponde con la primera fase de un proceso de adaptación a un nuevo contexto socio-sanitario en España.

\section{Las influencias externas marcan la incorporación de la Medicina de Familia en la Universidad}

Sin lugar a dudas la creación de la Especialidad Médica de Medicina de Familia y Comunitaria y su incorporación al sistema de formación médica de postgrado por el sistema de residencia en el seno del sistema sanitario español (sistema MIR - Médicos Internos y Residentes) en 1978 constituye el origen de un movimiento, nacido extramuros de las Facultades de
Medicina, que destaca el papel del ámbito extrahospitalario en la enseñanza/aprendizaje de la medicina. La duración del programa de formación en la Especialidad de Medicina de Familia es en la actualidad de 3 años, con estancia tanto en el Hospital como en el Centro de Salud.

Con ocasión de la Ley General de Sanidad de 1986, pieza básica de la reforma sanitaria en España, se incorpora la obligación que tienen las Facultades de incorporar a su estructura docente al menos a 3 centros de salud. Esta norma no empieza a cumplirse hasta diez años después y aún existen algunas Facultades en España que no la cumplen.

Desde 1995 y por acuerdo de todos los países de la Unión Europea, para acceder al puesto de Médico de Familia/Médico General en el sistema público es necesario estar en posesión del título de postgrado correspondiente. En una primera fase, en las Facultades de Medicina se produjo cierto rechazo a esta idea de formación de postgrado, y que esta fuera la Especialidad de Medicina Familiar y Comunitaria por via MIR, obligatoria para acceder al puesto de Médico de Familia, ya que el terminar la carrera de 6 años no era suficiente para incorporarse a ningún puesto de trabajo en el sistema sanitario público, a diferencia de lo que había sucedido con las promociones anteriores a 1995.

A pesar de la conocida resistencia al cambio que muestra la rígida estructura universitaria, paso a paso se van aceptando e incorporando a los nuevos planes de estudio distintas actividades que suponen la presencia de los estudiantes de Medicina al lado de los Médicos de Familia en los Centros de Salud. 


\section{Las nuevas actividades de Medicina de Familia en la Universidad}

Los programas específicos de Medicina de Familia y Atención Primaria no son idénticos entre las distintas Facultades que los han ido incorporado a sus "curricula", pero existe un común denominador a la mayoría de ellos: se tratan de actividades eminentemente prácticas basadas en la estancia durante 2-4 semanas del estudiante de los últimos años de carrera en un Centro de Salud bajo la tutoría específica de un Médico de Familia y de asignaturas optativas que también incorporan un componente practico importante ${ }^{(2)}$.

Como ejemplo se cita el programa actual de la Facultad de Medicina de la Universidad Autónoma de Madrid. Se trata de tres actividades diferentes:

- ROTACIÓN en un Centro de Salud, obligatoria para todos los alumnos de sexto curso, de un mes de duración. Cada alumno es asignado a un Médico de Familia y participa de todas las actividades que este realiza en el Centro o en la visita al domicilio de los pacientes.

- CONTACTO PRECOZ CON EL PACIENTE EN ATENCIÓN PRIMARIA. Se trata de una asignatura optativa para los alumnos de tercer curso, equivalente a 6 créditos. Es una actividad que supone la realización de un trabajo de campo sobre un paciente concreto y su entorno familiar, laboral y de vivienda, con el objetivo de captar la multifactorialidad en el proceso de salud/enfermedad así como el papel del médico en el sistema sanitario. Es una actividad exclusivamente practica y directamente tutorizada por un Médico de Familia.

- ATENCIÓN PRIMARIA: LA IMPORTANCIA DE LA MEDICINA DE FAMILIA. Se trata de una asignatura optativa para los alumnos de quinto o sexto curso, de 6 créditos, que supone el seguimiento de un programa de clases y la elaboración de un trabajo práctico. Todas las clases y el trabajo práctica están a cargo de los médicos de familia de los Centros de Salud adscritos a la Unidad de Atención Primaria de la Facultad de Medicina.

\section{El proceso de consolidación de la Medicina de \\ Familia en la enseñanza de pregrado de la medicina es lento pero inevitable}

A pesar de que en la actualidad la incorporación de estas nuevas actividades docentes se pueden situ- ar mas como una respuesta de adaptación y seguimiento que como una actitud real de cambio y liderazgo por parte de la universidad, la tendencia apunta en la dirección de la aceptación definitiva de la Medicina de Familia en el programa de pre-grado de las Facultades de Medicina. Esta aceptación será evidente cuando la medicina de familia se incorpore a la estructura universitaria, con el reconocimiento de profesores titulares y de un área de conocimiento específica. Pero este es un camino largo.

Hoy día, entre el profesorado numerario, se valora la incorporación de actividades docentes realizadas en el ámbito de la atención primaria pero en el seno de las asignaturas clásicas. A los médicos de familia se les reconoce como buenos profesores de clases prácticas pero sin reconocerles todavía un protagonismo real en el liderazgo de las nuevas tendencias en la enseñanza/aprendizaje de la Medicina. La orientación de la enseñanza hacia la comunidad aún no está suficientemente asumida por un cuerpo docente aún muy centrado en el hospital como el lugar básico de enseñanza de la medicina.

El cambio de las patologías prevalentes y de los casos que son vistos y tratados fuera del Hospital influirá, aún desde la orientación mas biologicista y centrada en la enfermedad, en el reconocimiento de un papel cada vez mayor del Centro de Salud como lugar imprescindible para el aprendizaje del estudiante de medicina.

Poco a poco las Unidades de Atención Primaria en las Facultades de Medicina españolas irán consolidando su estructura y su protagonísmo en el desarrollo del currículum de pregrado.

\section{BIBLIOGRAFÍA}

1 - GRUPO DE TRABAJO DE PREGRADO. La medicina de familia como área de conocimiento. Serie Documentos. Sem FYC, no 10. Barcelona, 1998

2 - BONAL P; GIL V; MARTIN A \& PINTO A. La medicina de familia como área de conocimiento. Aten Primaria 23: 151-174, 1999. 